\title{
Parental stress before, during, and after pediatric stem cell transplantation: a review article
}

\author{
C. M. J. Vrijmoet-Wiersma • R. M. Egeler • \\ H. M. Koopman • A. Lindahl Norberg • \\ M. A. Grootenhuis
}

Received: 16 December 2008 / Accepted: 18 June 2009/Published online: 2 July 2009

(C) The Author(s) 2009. This article is published with open access at Springerlink.com

\begin{abstract}
Goals of work Pediatric stem cell transplantation (SCT) is a stressful treatment for children with relapsed or high-risk malignancies, immune deficiencies and certain blood diseases. Parents of children undergoing SCT can experience ongoing stress related to the SCT period. The aim of this article was to present a literature review of articles on parental distress and adaptation before, during, and after SCT and to identify risk and protective factors.

Materials and methods The review was conducted systematically by using PubMed, Web of Science, PsychInfo, and Picarta databases. Eighteen articles met our inclusion criteria: publishing date between January 1, 1990 and January 1, 2009; studies concerning parents of children undergoing SCT; studies examining the psychological adjustment and/or stress reactions of parents as primary outcomes and studies available in English.

Main results Highest levels of parental stress are reported in the period preceding SCT and during the acute phase.
\end{abstract}

C. M. J. Vrijmoet-Wiersma $(\bowtie) \cdot$ R. M. Egeler

Pediatric Department, Leiden University Medical Center,

P.O. Box 9600, 2300 RC Leiden, The Netherlands

e-mail: c.m.j.vrijmoet-wiersma@lumc.nl

H. M. Koopman

Medical Psychology, Leiden University Medical Center,

Leiden, The Netherlands

\section{A. L. Norberg}

Childhood Cancer Research Unit,

Department of Woman and Child Health, Karolinska Institutet,

Stockholm, Sweden

\section{A. Grootenhuis}

Pediatric Psychosocial Department, Emma Children's Hospital, Academic Medical Center Amsterdam,

Amsterdam, The Netherlands
Stress levels decrease steadily after discharge in most parents. However, in a subgroup of parents, stress levels still remain elevated post-SCT. Parents most at risk in the longer term display highest levels of stress during the acute phase of the SCT.

Conclusions Psychosocial assessment before SCT, during the acute phase and in the longer term, is necessary to identify parents in need for support and follow-up care.

Keywords Pediatric SCT · Parental stress · Adaptation · Review

\section{Introduction}

Stem cell transplantation (SCT) is an invasive treatment for seriously ill children who have hematological, oncological, or metabolical diseases. Recently, for some high-risk leukemia protocols, SCT has become close to being a first-choice treatment. SCT is a perilous treatment, associated with significant mortality and morbidity [5]. It involves a lengthy hospital admission in an isolated environment to prevent infections and treatment with high doses of chemotherapy and/or radiation, followed by infusions of donor stem cells [22]. During the acute phase of SCT, children report high levels of somatic distress, mood disturbance [30], nausea and pain [7], and fatigue and malaise [29]. In the first 4-6 months post-SCT, children are still susceptible to infections and need to live with restrictions. SCT has a profound impact on the lives of children and parents, both during the acute phase and afterwards. Parents are faced with the need to provide both physical and emotional care for their ill child during a long and stressful period. Furthermore, they have to deal with their own emotions, especially with the realistic fear of 
losing their child and they have to make complicated decisions about the treatment together with the multidisciplinary team. Some parents are also faced with supporting one of their other children who will be acting as a sibling donor and increasingly, parents are acting as haploidentical donors themselves in case no appropriate donor has been found.

Despite improved survival rates, SCT remains a highrisk procedure. The result of the transplantation depends on several risk factors, including type and status of the underlying disease [5]. After treatment, parents and children are faced with the risk of recurrence, acute or chronic graft-versus-host disease, and numerous possible late effects such as pulmonary disease, growth problems, and infertility $[4,21]$. Even in the longer term, the child's illness and the SCT may influence parents' everyday lives [8]. SCT treatment protocols have changed in the past decades, one of the most important differences between treatment now and in the 1990s is the shortened admission period. On the one hand this is an improvement because parents may have fewer concerns about the practical issues during admission, e.g. being away from home for a long period and dealing with work-related stress. On the other hand, caring for a child at home post-SCT can be a heavy burden on parents and families. In addition, increasing survival rates entail increasing numbers of survivors with possible long-term side effects. Moreover, the fear of losing the child is still realistic. Accordingly, changed treatment protocols may not make any difference for parental stress levels.

The field of parental adaptation to their child's SCT has gained more attention in the past decade; most studies have been conducted in the past 8 years $[18,31]$. The majority of studies have focused on parental stress and adjustment preSCT, during the acute phase, and 3, 6, or 18 months postSCT, e.g. $[5,18]$. These time points seem to cover the SCT time frame well: 3 months post-SCT, many children still suffer from the after effects of the SCT, whereas after 6 months, most of the children can return to school and pick up their old lives, even if their health is suboptimal [1]. Twelve to eighteen months post-SCT, the majority of children report to have a health-related quality of life (HRQoL) comparable to healthy peers $[2,7]$. However, certain aspects of HRQoL seem to be affected in the long-term in many survivors, for example cognitive functions and pain, which has been demonstrated recently by Löf et al. [15].

No review articles have been published in this specific area so far. In related areas, however, review studies have focused on adjustment and coping of parents of children with cancer $[9,40]$; on the quality of life and/or emotional adjustment of children after SCT $[2,38]$; on the psychological adjustment of families of adult SCT patients [14] and on the psychosocial impact of SCT of adult patients $[11,23]$. The aim of our article was to conduct a systematic review of the current literature (1990 to 2008) on parental distress and adaptation to their child's SCT and to identify risk and protective factors.

\section{Materials and methods}

Search strategy for identification of studies

Several research engines were used to obtain the studies included in this review: Pubmed, Web of Science, PsychInfo, and Picarta. These databases were searched for one of the words: BMT, bone marrow transplantation, SCT, or stem cell transplantation combined with the following words used in headings, keywords, subjects, or abstracts: pediatric, paediatric, parent, child, mother, father, AND/OR stress, distress, post-traumatic stress disorder (PTSD), posttraumatic stress symptoms (PTSS), anxiety, depression, parental stress, parental distress, psychological, adjustment, and coping. Subsequently, reference lists of the relevant articles were examined to identify additional papers that met the search criteria and a hand search was conducted in several relevant academic journals.

Criteria for considering studies for the review

Criteria for inclusion were: publishing date between January 1, 1990 and January 1, 2009; studies concerning parents of children undergoing SCT; studies examining the psychological adjustment and/or stress reactions of parents as a primary outcome; and studies available in English. Exclusion criteria were: reviews, guidelines, protocols, commentaries, and other descriptive articles; studies focusing on psychological adjustment of pediatric SCT patients only; studies focusing on other critical illnesses or including other treatments; and studies focusing solely on intervention programs.

\section{Description of the studies}

Eighteen studies were selected for this review. Table 1 contains a descriptive summary of the articles. We included the aim of the study, number and characteristics of parents and children, methodological features (e.g. study design, timing of measurement); measures; and main results. The indications for SCT varied among the different studies. Most studies reported the underlying diagnosis, type of transplant and type of donor, transplant risk, and disease risk category [17]. On average $80 \%$ of the children undergoing SCT suffered from a malignant disease and around $60 \%$ of the children in the studies underwent allogeneic SCT (transplantation with bone marrow from a foreign donor) as opposed to autologous SCT (transplantation with own body material). 


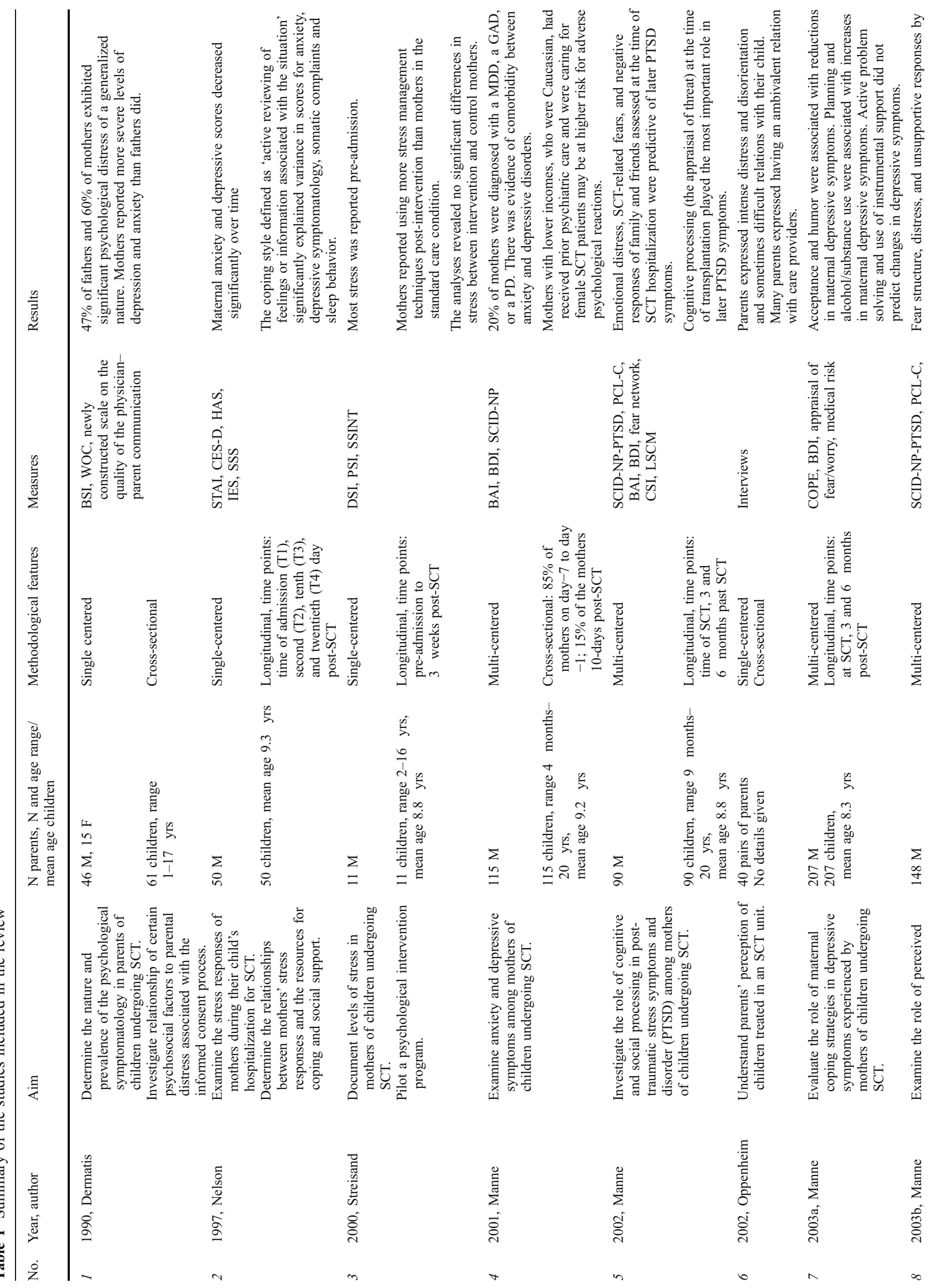




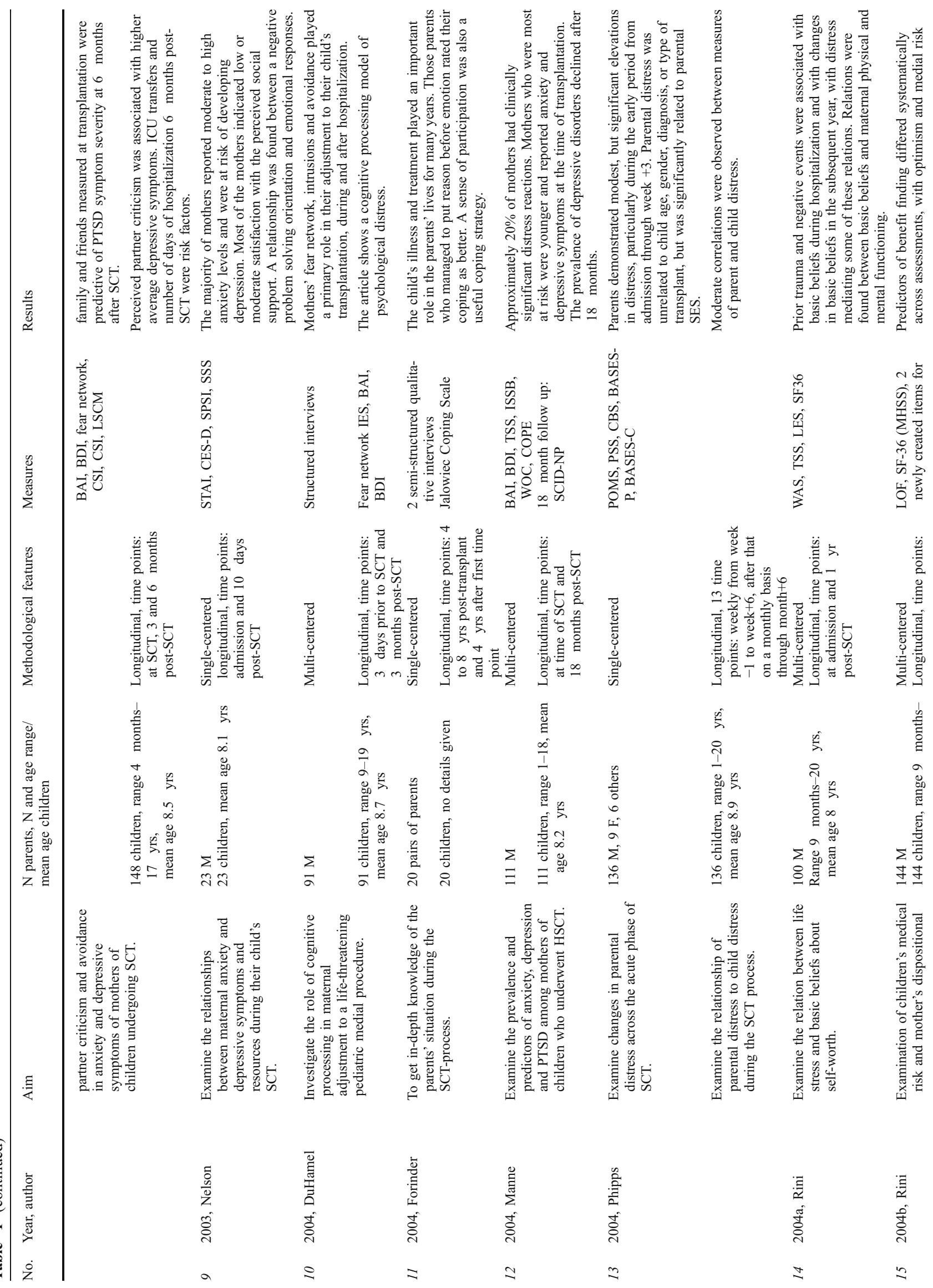



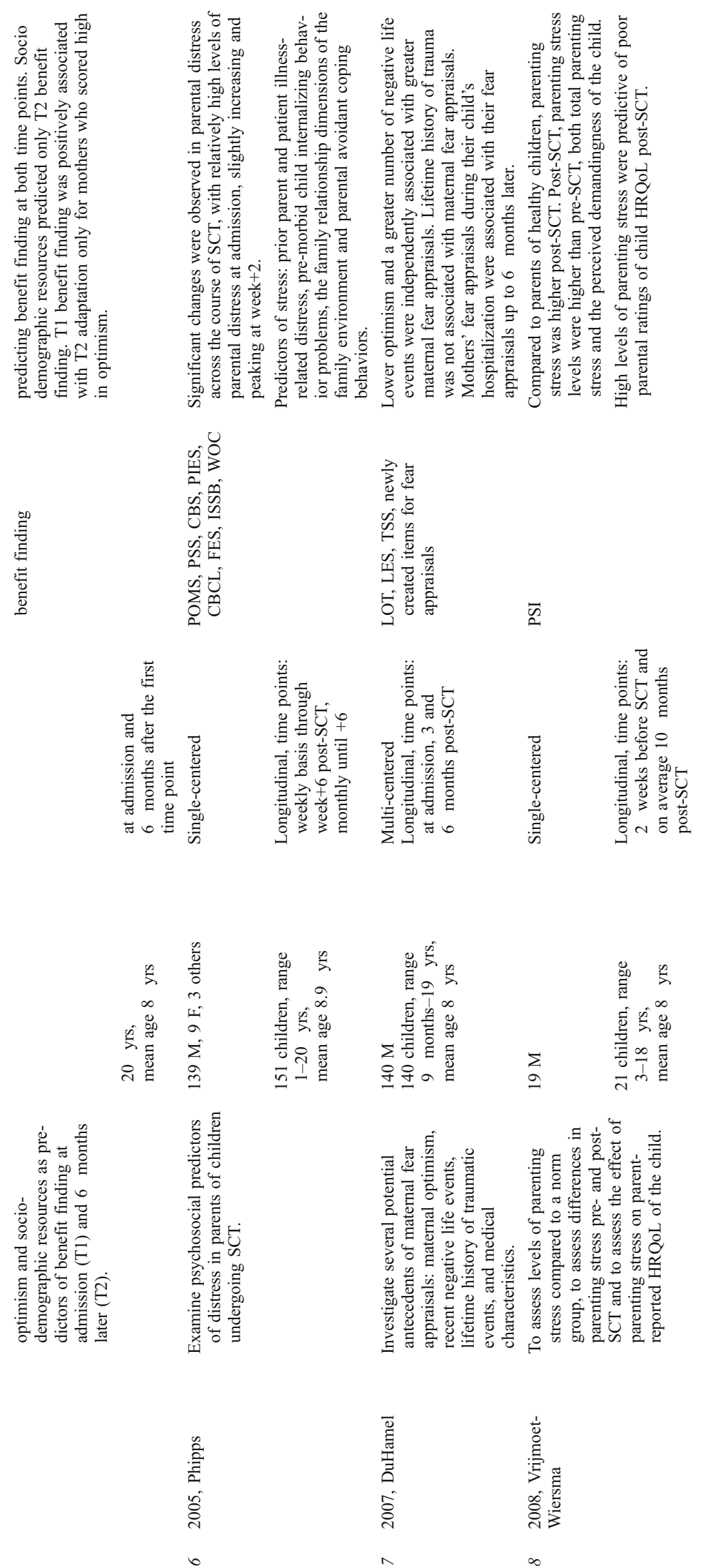

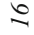

$\approx$

$\infty$

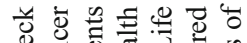

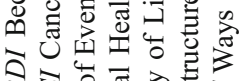

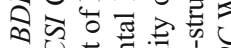

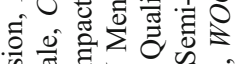

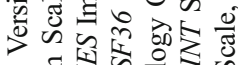

믈 은

ญ

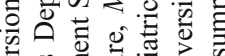

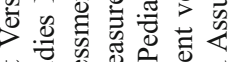

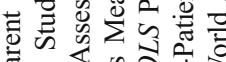

幽.

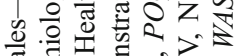

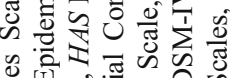

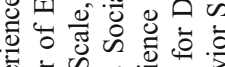

过

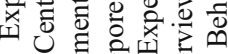

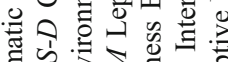

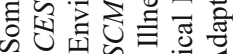

들 군

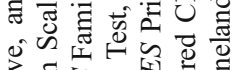

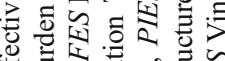

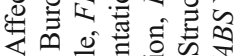

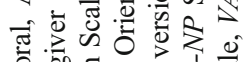

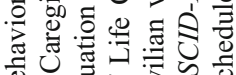

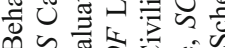

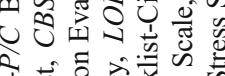

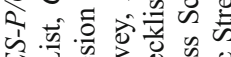

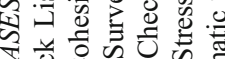

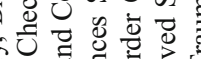

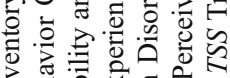

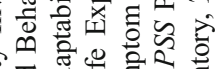

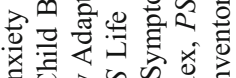

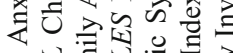

委

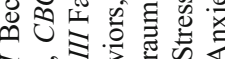

상

of o

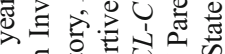

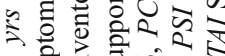

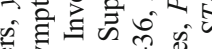

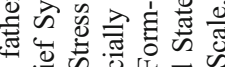

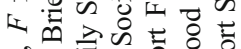

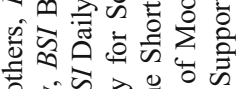

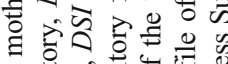

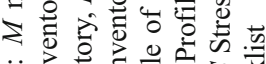

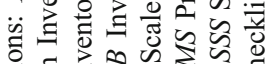

을

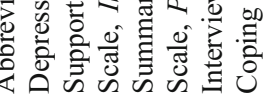


The studies in our review did not distinguish between the experiences of parents of children undergoing SCT once and parents of children who had multiple SCT experiences, who will undoubtedly have to face a unique set of stressors.

In this review article, we will first discuss the methodological qualities of the studies, next we will summarize the main results of the studies, and lastly we will discuss risk and protective factors of parental adaptation to their child's SCT.

\section{Methodological qualities of the selected studies}

Study design and timing of assessment

Three of the included studies used a cross-sectional study design $[3,20,27]$ and one study was descriptive/retrospective [8]. The other 14 studies used a prospective longitudinal design with repeated measures, ranging from two to 13 measurement points. However, only a few of these studies followed a particular aspect of parental distress over time, e.g. $[18,32]$. Exactly half of the studies included in the review used a multi-centered design, the other nine studies recruited participants from one medical center. Until now, only one intervention study has been published in this area. It included mothers of children undergoing SCT [36] and was based on a stress inoculation model.

In most longitudinal studies, two measurement points were used. The first time point was between 47 to 1 day(s) pre-admission and a few days post-SCT. The time point for the second assessment varied strongly between the studies, ranging from 1 week post-SCT to 18 months post-SCT. Phipps et al. [31, 32] used up to 13 time points in both studies. Only one of the studies assessed long-term parental distress, 4 to 8 years after stem cell transplantation [8].

\section{Participants}

The majority of the studies (13 in total) used only mothers as respondents. Sample size in the studies varied from 11 [36] to 207 parents [18]. Eleven of the studies included more than 90 parents. All studies described the recruitment process. Phipps et al. [31, 32] assessed one of the caregivers, resulting in $90 \%$ mothers. In only three studies [3, 27, 39], both parents were used as respondents. The age range of the children was most often 1 to 20 years of age with a mean age at first assessment between 8 and 9 years. In two studies, the age of the children was not mentioned $[8,27]$.

\section{Outcome measures}

The conceptualization of stress or distress varied widely between the studies. The distinction between the assess- ment of (subclinical) levels of distress on the one hand and clinical psychiatric diagnoses was not always clearly made, which makes comparisons difficult. Anxiety and depression were studied as manifestations of parental distress in nearly all of the studies. Other manifestations of parental distress or psychiatric disorders were disturbed and obsessivecompulsive thinking [3], post-traumatic stress symptoms $[16,17]$, generalized anxiety disorder (GAD), panic disorder (PD), and major depressive disorder (MDD) [20]. Somatic complaints and changes in sleep behavior were added by some researchers $[25,34,35]$ as symptoms of parental distress. Variables influencing parental stress levels were mostly operationalized as 'coping' [18, 24, 25, 32], 'family functioning' [32], 'parenting stress issues' [39], and 'social support', both positively and negatively perceived $[19,25,32]$.

In most studies multiple measures were used, most often self-report questionnaires combined with, or additional to, interviews as a way of collecting data. In the majority of studies standardized questionnaires were used to assess parental distress reactions (e.g. Beck Depression Inventory (BDI), Beck Anxiety Inventory (BAI), State-Trait Anxiety Inventory (STAI), and Parental Stress Index (PSI)), e.g. [17, 20]. Disease- or context-specific measures were used less frequently. Phipps et al. [31, 32] developed and used the Prior Illness Experiences Scale (PIES) to assess previous parent and child experiences with cancer therapy and inpatient hospitalization. Rini et al. [35] created two items to measure benefit finding in their study and DuHamel and her study group developed a scale to assess maternal fear appraisals [6].

\section{Results}

Parental stress

Feelings of anxiety and depression, post-traumatic stress symptoms, disturbed or obsessive compulsive thinking, and somatic complaints are the most common stress reactions parents report before, during, and after SCT. Parental stress levels were reported to be higher than norm groups pretransplant $[3,32,36]$ and during the acute phase of the SCT, peaking at 2-3 weeks post-SCT [32]. The most common observation was that parental distress levels decreased over time after SCT $[5,36]$, with the sharpest declines between 3 6 months post-transplant [28]. A recent study, however, showed that parents, on average 10 months post-SCT, reported higher parenting stress levels and, specifically, felt less competent as a parent [39]. In the longer term, 4 to 8 years post-SCT [8], many parents reported that their child's illness and subsequent treatment played an important role in their lives for years, ranging from parents still struggling on a 
daily basis to parents who put this ordeal more or less behind them. Studies comparing mothers and fathers are few in this area, since most studies included mothers only. Dermatis and Lesko [3] found higher levels of depression and phobic anxiety in mothers than in fathers.

Table 1 depicts the results of the 18 studies included in the review.

\section{Risk and protective factors}

Many factors have been identified to influence levels of parental distress. The most frequently identified risk factor for parental distress in the longer term is the manner in which the parent is able to handle stress during the acute phase. We grouped the risk factors into three clusters, based on a manual count of the determinants described in the included studies:

1. Disease factors, i.e. if the child had been transferred to the ICU and if it had had a higher number of hospitalizations 6 months post-SCT, parents reported more anxious and depressive symptoms [19]. Higher transplant risk was also associated with higher parental distress during the child's post hospital stage of recovery [6].

2. Psychological factors and parental coping: mother's appraisal of threat to her child's life [5, 16], a greater number of negative life events [6], prior parent and patient experiences of distress associated with the child's illness [32], avoidance and intrusions [18, 32], alcohol/substance abuse [18], perceived partner criticism [19], and an unsupportive family environment [32] all added to parental (i.e. mostly maternal) stress levels. Furthermore, mothers experiencing depressive symptoms during the acute SCT phase had a higher probability to be diagnosed with a psychiatric disorder 18 months post-SCT [17]. Parent distress has also been associated with child distress: child mood disturbance at admission was predictive of parent global distress over time [28].

3. Socio-demographic risk factors: younger maternal age [17] and lower social economical status (SES) [31] were associated with higher levels of stress throughout the SCT process.

Factors that did not appear to influence parental stress were: the age of the child, type of diagnosis, type of transplant, or the nature of the germ-free environment in which the child was placed $[3,5,32]$. Other objective medical aspects of the child's condition (i.e. disease risk, treatment course, and current disease status) than the factors mentioned under the first cluster (see above) were not related to parental stress levels or fear appraisals. It seems that the subjective appraisal of these factors is a better predictor of parental stress than the objective disease characteristics [6].

Protective factors or predictors of good psychological adaptation have been identified in terms of 'benefit finding' [35]: mothers who were optimistic by nature reported most benefit finding both during the acute SCT phase and 6 months later. Benefit finding is defined as 'an attempt to restore positive basic beliefs about the self and the world that have been challenged by a traumatic experience' $[35$, 37]. Acceptance and humor as coping mechanisms were associated with reductions in maternal depressive symptoms [18] and 'putting reason before emotion' was identified as another coping mechanism associated with positive outcomes [8]. Lastly, a supportive family environment was associated with lower distress levels throughout the transplant process [32].

\section{Discussion}

Having a child undergo stem cell transplantation is a stressful event for any parent. Feelings of anxiety and depression, post-traumatic stress symptoms, disturbed or obsessive compulsive thinking, and somatic complaints are the most common stress reactions parents report before, during, and after SCT. The process of SCT is comprised of several phases and distress levels seem most elevated in the pre-SCT phase and the acute phase during hospitalization, but can stay elevated after discharge. Most parents return to healthy levels of psychosocial functioning 18 months postSCT, but a subset of parents reports persistent symptoms years later in terms of anxiety, depressive feelings, and post-traumatic stress symptoms (arousal, avoidance, and recurring memories). Certain maternal coping strategies (e. g. acceptance, humor, putting reason before emotion, and having positive cognitive appraisals) during the acute phase of SCT have been identified as protective factors.

It has been shown that dispositional factors and prior experiences influence the way an individual appraises an event such as SCT and adjusts to it. For example, mothers who were more optimistic by nature reported lower fear appraisals at the time of their child's hospitalization for SCT [6] and later on, post-SCT. Optimism seems to be a more or less stable trait that can serve as a coping mechanism and a buffer [10], like positive reframing [18] and benefit finding [35]. Mothers who have encountered more negative life events in the months before their child's SCT reported more fear appraisals. It is hypothesized that traumatic events can trigger increased arousal, cognitions that one's life is difficult and traumatic [17], and a tendency to interpret potentially harmful new events more negatively [6]. Pre-existing factors should be assessed and used as starting points for psychosocial interventions. 
The present review reveals potential areas of improvement in future research. In the 18 studies included in this review a variety of definitions of the core elements of the psychological stress process have been used, often described together and simply referred to as 'stress'. It is important to clarify what is meant by 'stress' and to specify both the temporal course of a stressor [13] and to identify SCT-specific stressors. Post-traumatic stress reactions imply an existential challenge, but findings suggest that the complex situation of SCT involves several different stressors for the parents. To facilitate communication and collaboration it is necessary to be more specific in the terminology used to describe the psychological reactions of both parents and patients and to make a clear distinction between stress as a predictor variable and psychological stress as an outcome.

In most of the research in this area, no distinction was made between subclinical manifestations of parental distress versus psychiatric states. This is unfortunate, because in the latter approach, parents tend to be 'pathologized' [33] instead of assuming that the majority of families with a seriously ill child are competent and adaptively organized families, without any elevations in their a priori risk (as a group) for psychopathology [12]. Furthermore, it seems that in multidisciplinary SCT teams often there is no consensus of what is 'normal' distress or 'adequate coping' in this context. For example, young and inexperienced nurses can get worried about a parent in tears whereas an experienced social worker or psychologist may feel that a certain level or manifestation of distress is 'normal'. This issue points to the need for adequate psychosocial screening by pediatric psychologists pre-admission and during the acute phase of SCT, in order to target those parents most in need for psychosocial guidance and intervention.

Family functioning, an area of increasing importance in the pediatric psychology literature, is still understudied in parents of children undergoing SCT. The experience of fathers is another area of neglect. In many studies on parental stress of parents of pediatric cancer patients, higher stress levels have been found for mothers than for fathers [40], but recent research has shown that the experience of the child's illness often is as stressful for fathers as for mothers [26]. This finding points to the need to include fathers in future studies.

Strengths of the studies included in our review are the large number of longitudinal designs and multi-centered studies and the majority of studies with 90 participants or more. We have found only a minority of studies in which disease- or contextspecific measures were used and this is unfortunate, because $\mathrm{SCT}$ is a highly complex treatment with very specific issues to deal with for parents. A combination of generic and diseasespecific instruments could further our understanding of parental distress trajectories during the course of the SCT.

\section{Conclusions}

The authors conclude that the majority of parents of pediatric SCT patients are resilient, 18 months post-SCT and beyond. The most frequently identified risk factor for parental distress in the longer term is the manner in which the parent is able to handle stress during the acute phase. Parents (mostly mothers) with the most severe stress reactions and fear appraisals during the acute phase, continue to experience heightened levels of anxiety, depressive symptoms, and PTSS later on.

The next step is to develop and systematically examine feasible, limited, brief interventions for sub-clinical manifestations of psychological distress prior to and during the acute phase of SCT in parents who have been identified as 'risk' group. Follow-up care is needed for parents, especially when their child recovers and when control visits to the hospital become less frequent. Intervention research is a growing area in medical psychology and despite the many methodological challenges, efforts should be made to implement and evaluate existing intervention programs in this parent group. This can only be done through sound SCT-specific-assessment, well-funded (inter) national cooperation, and well-developed study designs. Lastly, the ethical domain of conceiving designed children as donors is an area of interest that deserves be studied in the future, as well as the issue of stress in parents of children who need to undergo more than one stem cell transplantation or whose children suffer from more serious late effects, such as chronic graft-versus-host disease or other health problems.

Acknowledgements We would like to thank our psychology master student Saskia de Ridder for her contribution to the literature search and for setting up the table and we owe Robbert Bredius thanks for his important comments on the manuscript.

Open Access This article is distributed under the terms of the Creative Commons Attribution Noncommercial License which permits any noncommercial use, distribution, and reproduction in any medium, provided the original author(s) and source are credited.

\section{Reference}

1. Barrera M, Boyd-Pringle LA, Sumbler K, Saunders F (2000) Quality of life and behavioral adjustment after pediatric bone marrow transplantation. Bone Marrow Transplant 26(4):427435

2. Clarke SA, Eiser C, Skinner R (2008) Health-related quality of life in survivers of BMT for paediatric malignancy: a systematic review of the literature. Bone Marrow Transplant 42:73-80

3. Dermatis H, Lesko LM (1990) Psychological distress in parents consenting to child's bone marrow transplantation. Bone Marrow Transplant 6(6):411-417 
4. Duell T, van Lint MT, Ljungman P, Tichelli A, Socie G, Apperley JF et al (1997) Health and functional status of long-term survivors of bone marrow transplantation. Ann Intern Med 126 (3):184-192

5. DuHamel KN, Manne S, Nereo N, Ostroff J, Martini R, Parsons S et al (2004) Cognitive processing among mothers of children undergoing bone marrow/stem cell transplantation. Psychosom Med 66(1):92-103

6. DuHamel KN, Rini C, Austin J, Ostroff J, Parsons S, Martini R et al (2007) Optimism and life events as predictors of fear appraisals in mothers of children undergoing hematopoietic stem cell transplantation. Psychooncology 16(9):821-833

7. Felder-Puig R, di Gallo A, Waldenmair M, Norden P, Winter A, Gadner $\mathrm{H}$ et al (2006) Health-related quality of life of pediatric patients receiving allogeneic stem cell or bone marrow transplantation: results of a longitudinal, multi-center study. Bone Marrow Transplant 38(2):119-126

8. Forinder U (2004) Bone marrow transplantation from a parental perspective. J Child Health Care 8(2):134-148

9. Grootenhuis MA, Last BF (1997) Adjustment and coping by parents of children with cancer: a review of the literature. Support Care Cancer 5(6):466-484

10. Grootenhuis MA, Last BF (1997) Predictors of parental emotional adjustment to childhood cancer. Psychooncology 6(2):115-128

11. Hoodin F, Weber S (2003) A systematic review of psychosocial factors affecting survival after bone marrow transplantation. Psychosomatics 44(3):181-195

12. Kazak AE, Rourke MT, Alderfer MA, Pai A, Reilly AF, Meadows AT (2007) Evidence-based assessment, intervention and psychosocial care in pediatric oncology: a blueprint for comprehensive services across treatment. J Pediatr Psychol 32 (9):1099-1110

13. La Greca AM, Lemanek KL (1996) Assessment as a process in pediatric psychology. J Pediatr Psychol 21(2):137-151

14. Lesko LM (1994) Bone marrow transplantation: support of the patient and his/her family. Support Care Cancer 2(1):35-49

15. Löf C, Winiarski J, Giesecke A, Ljungman P, Forinder U (2009) Health-related quality of life in adult survivors after paediatric allo-SCT. Bone Marrow Transplant 43(6):461-468

16. Manne S, DuHamel K, Nereo N, Ostroff J, Parsons S, Martini R et al (2002) Predictors of PTSD in mothers of children undergoing bone marrow transplantation: the role of cognitive and social processes. J Pediatr Psychol 27(7):607-617

17. Manne S, DuHamel K, Ostroff J, Parsons S, Martini DR, Williams SE et al (2004) Anxiety, depressive, and posttraumatic stress disorders among mothers of pediatric survivors of hematopoietic stem cell transplantation. Pediatrics 113(6):1700-1708

18. Manne S, DuHamel K, Ostroff J, Parsons S, Martini DR, Williams SE et al (2003) Coping and the course of mother's depressive symptoms during and after pediatric bone marrow transplantation. J Am Acad Child Adolesc Psych 42(9):1055-1068

19. Manne S, DuHamel K, Winkel G, Ostroff J, Parsons S, Martini R et al (2003) Perceived partner critical and avoidant behaviors as predictors of anxious and depressive symptoms among mothers of children undergoing hemopoietic stem cell transplantation. J Consult Clin Psychol 71(6):1076-1083

20. Manne S, Nereo N, DuHamel K, Ostroff J, Parsons S, Martini DR et al (2001) Anxiety and depression in mothers of children undergoing bone marrow transplant: symptom prevalence and use of the Beck depression and Beck anxiety inventories as screening instruments. J Consult Clin Psychol 69(9):1037-1047

21. Matthes-Martin SLM, Ladenstein R, Emminger W, Felsberger C, Topf R, Gadner H et al (1999) Organ toxicity and quality of life after allogeneic bone marrow transplantation in pediatric patients: a single center retrospective analysis. Bone Marrow Transplant 23 (10):1049-1053
22. Mosher CE, Redd W, Rini C, Burkhalter JE, DuHamel KN (2009) Physical, psychological, and social sequelae following hematopoietic stem cell transplantation: a review of the literature. Psychooncology 18:113-127

23. Neitzert CS, Ritvo P, Dancey J, Weiser K, Murray C, Avery J (1998) The psychosocial impact of bone marrow transplantation: a review of the literature. Bone Marrow Transplant 22(5):409-422

24. Nelson AE, Gleaves L, Nuss S (2003) Mothers' responses during the child's stem cell transplantation: pilot study. Pediatr Nurs 29 (3):219-223

25. Nelson AE, Miles MS, Belyea MJ (1997) Coping and support effects on mothers' stress responses to their child's hematopoietic stem cell transplantation. J Pediatr Oncol Nurs 14(4):202-212

26. Norberg AL, Boman K (2008) Parent distress in childhood cancer: a comparative evaluation of posttraumatic stress symptoms, depression and anxiety. Acta Oncol 47(2):267-274

27. Oppenheim D, Vasselon S, Hartmann O (2002) How do parents perceive high-dose chemotherapy and autologous stem cell transplantation for their children. Bone Marrow Transplant 30(1):35-39

28. Phipps S (2006) Psychosocial and behavioral issues in stem cell transplantation. In: Brown RT (ed) Comprehensive handbook of childhood cancer and sickle cell disease, a biopsychosocial approach. Oxford University Press, New York

29. Phipps S, Dunavant M, Garvie PA, Lensing S, Rai SN (2002) Acute health-related quality of life in children undergoing stem cell transplant: I. Descriptive outcomes. Bone Marrow Transplant 29(5):425-434

30. Phipps S, Dunavant M, Lensing S, Rai SN (2002) Acute healthrelated quality of life in children undergoing stem cell transplant: II. Medical and demographic determinants. Bone Marrow Transplant 29(5):435-442

31. Phipps S, Dunavant M, Lensing S, Rai SN (2004) Patterns of distress in parents of children undergoing stem cell transplantation. Pediatric Blood \& Cancer 43(3):267-274

32. Phipps S, Dunavant M, Lensing S, Rai SN (2005) Psychosocial predictors of distress in parents of children undergoing stem cell or bone marrow transplantation. J Pediatr Psychol 30(2):139-153

33. Quittner AL, Davis MA, Modi AC (2003) Health-related quality of life in pediatric populations. In: Roberts MC (ed) Handbook of pediatric psychology, 3rd edn. Guilford, New York, pp 696-709

34. Rini C, Manne S, DuHamel KN, Austin J, Ostroff J, Boulad F et al (2004) Changes in mothers' basic beliefs following a child's bone marrow transplantation: the role of prior trauma and negative life events. J Trauma Stress 17(4):325-333

35. Rini C, Manne S, DuHamel KN, Austin J, Ostroff J, Boulad F et al (2004) Mothers' perceptions of benefit following pediatric stem cell transplantation: a longitudinal investigation of the roles of optimism, medical risk, and sociodemographic resources. Ann Behav Med 28(2):132-141

36. Streisand R, Rodrigue JR, Houck C, Graham-Pole J, Berlant N (2000) Brief report: parents of children undergoing bone marrow transplantation: documenting stress and piloting a psychological intervention program. J Pediatr Psychol 25(5):331-337

37. Taylor SE (1983) Adjustment to threatening events: a theory of cognitive adaptation. Am Psychol 38:1161-1173

38. Tsimicalis A, Stinson J, Stevens B (2005) Quality of life of children following bone marrow transplantation: critical review of the research literature. Eur J Oncol Nurs 9(3):218-238

39. Vrijmoet-Wiersma CMJ, Kolk AM, Grootenhuis MA, Spek EM, van Klink JMM, Egeler RM et al (2009) Child and parental adaptation to pediatric stem cell transplantation. Support Care Cancer 17(6):707-714

40. Vrijmoet-Wiersma CMJ, van Klink JMM, Kolk AM, Koopman HM, Ball LM, Maarten Egeler R (2008) Assessment of parental psychological stress in pediatric cancer: a review. J Pediatr Psychol 33(7):694-706 